Reprod. Nutr. Dévelop., 1987, 27 (1 B), 221-222.

\title{
Comparaison de deux types de marqueurs, terres rares et chrome mordancé, pour la mesure du temps de séjour des aliments dans les estomacs du ruminant
}

\author{
J. GONZALEZ $\left({ }^{1}\right)\left({ }^{*}\right)$, C. PONCET $\left({ }^{*}\right)$, Brigitte MICHALET-DOREAU $(*)$ \\ (*) Laboratoire des Aliments, \\ (**) Laboratoire de la Digestion des Ruminants, \\ I.N.R.A. Theix, 63122 Ceyrat, France.
}

Summary. Ytterbium-169 and cerium-141 were compared to chromium-mordanted fiber as markers of the transit rate of the particulate phase in sheep digesta. The retention times estimated using rare earths were always shorter than with chromium. The reasons for these differences have been discussed.

Les terres rares (TR) et le chrome mordancé, marqueurs de transit des particules alimentaires les plus employés aujourd'hui, ont rarement été utilisés simultanément pour permettre leur comparaison. Or, on sait qu'ils possèdent des caractéristiques différentes : la solidité de la fixation d'une TR sur un aliment, variable selon la nature biochimique du support et la technique de préparation rend possible son transfert sur d'autres particules; inversement, le $\mathrm{Cr}_{2} \mathrm{O}_{3}$ mordancé est indissociable de son support rendu totalement indigestible, à la différence du marquage par une TR. Nous avons comparé ces 2 techniques de marquage en les appliquant simultanément pour l'étude du transit digestif de 3 aliments.

Matériel et méthodes. Du tourteau de soja, de la luzerne déshydratee en pellets et du foin de dactyle haché ont été marqués, après extraction (lavage $1 \mathrm{~h}$ à $90{ }^{\circ} \mathrm{C}$ avec détergent du commerce) de leurs constituants rapidement solubles, soit par une TR radioactive - ytterbium $\left({ }^{169} \mathrm{Yb}\right)$ sur les 2 premiers aliments, cérium $\left({ }^{141} \mathrm{Ce}\right)$ sur le foin - soit par mordançage au chrome (respectivement 52 , 85 , et $89 \mathrm{mg} \mathrm{Cr}$ fixé $/ \mathrm{g}$ fibre sur le foin, la luzerne et le soja. Le marquage par la TR a été réalisé par trempage $(24 \mathrm{~h})$ suivi d'une extraction par l'acide citrique (Ellis et Beever, 1984). Le transit digestif dans les estomacs de chacun de ces aliments a été mesuré sur 3 moutons adultes munis d'une canule simple duodénale, par prélèvements à intervalles croissants $(1 \mathrm{~h}$ à $6 \mathrm{~h})$ de contenu pendant $120 \mathrm{~h}$ après la distribution, en mélange avec le premier repas $(8 \mathrm{~h})$, de 2 doses $(40 \mathrm{~g} / \mathrm{dose})$ de I'aliment testé apportant chacune l'un des 2 types de marqueurs. Les animaux ont reçu successivement, en 8 repas égaux $/ \mathrm{j}$, à raison de $40 \mathrm{~g} \mathrm{MS} / \mathrm{kg} \mathrm{P}^{0,75}, 2$ rations composées de foin $(F)$ de dactyle haché et d'aliment concentré $(C)$ en proportion variable $(F / C=90 / 10$ et $40 / 60)$. Le transit du foin a été étudié dans la ration à base de foin, celui du soja et de la luzerne dans la ration riche en concentrés. Les courbes de concentration des marqueurs dosés par spectrométrie gamma ont été analysées suivant le modèle de Grovum et Williams (1973) pour le calcul des temps de séjour dans le rumen $\left.1 / k_{1}\right)$ et dans la caillette $\left(1 / k_{2}\right)$, et du délai d'apparition du marqueur (TT). Les différences entre marqueurs ont été testées par une analyse de variance par couple.

Résultats et discussion. Quel que soit l'aliment étudié ou le type de ration dans lequel il est ajouté, le temps de séjour dans le rumen $\left(1 / k_{1}\right)$ calculé avec le

(1) E.T.S.I.A. de Madrid - Departamento de Nutricion Animal Universidad Politechnica de Madrid-Ciudad Universitaria 28040 Madrid - Espagne. 
chrome mordancé est toujours supérieur à celui calculé en utilisant une TR (tabl. 1). Les différences sont plus importantes avec les aliments riches en parois (foin et luzerne déshydratée) sous forme de particules moyennes à grosses, qu'avec le tourteau de soja constitué de fines particules. En conséquence, alors que les temps de séjour du soja et de la luzerne mesurés avec une TR, sont identiques, ils diffèrent nettement lorsqu'ils sont calculés en utilisant le chrome. Cependant, cet effet " marqueur " n'est pas toujours significatif (cas de la luzerne) en raison de la variabilité entre animaux élevée, caractéristique de toute mesure effectuée au niveau du rumen, mais surtout observée ici avec les résultats obtenus en utilisant le chrome. Le temps de séjour dans le $2^{\mathrm{e}}$ compartiment du modèle, habituellement identifié à la caillette, de même que le délai d'apparition du marqueur au niveau duodénal, mesuré avec incertitude, ne sont pas significativement différents selon le marqueur utilisé. En conséquence, le temps de séjour d'un aliment dans l'ensemble des estomacs est supérieur lorsqu'il est mesuré avec le chrome, mais les écarts, même importants, ne sont pas significatifs.

TABL. 1. - Influence du type de marqueurs - terres rares $\left({ }^{169} \mathrm{Yb}\right.$ ou $\left.{ }^{141} \mathrm{Ce}\right)$ ou chrome mordancé sur le temps de séjour (heures) de 3 aliments - foin de dactyle haché, tourteau de soja, luzerne déshydratée - dans les estomacs du ruminant. (Moyenne \pm écart-type des résultats obtenus sur 3 moutons).

\begin{tabular}{|c|c|c|c|c|c|c|}
\hline Ration & \multicolumn{2}{|c|}{ Foin de dactyle $(90 \%)$} & \multicolumn{4}{|c|}{ Foin de dactyle $(40 \%)+$ Aliment concentré $(60 \%)$} \\
\hline Aliments testés & \multicolumn{2}{|c|}{ Foin de dactyle } & \multicolumn{2}{|c|}{ Tourteau de soja } & \multicolumn{2}{|c|}{ Luzerne déshydratée } \\
\hline Marqueurs & ${ }^{141} \mathrm{Ce}$ & $\mathrm{Cr}$ & ${ }^{169} \mathrm{Yb}$ & $\mathrm{Cr}$ & ${ }^{169} \mathrm{Yb}$ & $\mathrm{Cr}$ \\
\hline $\begin{array}{l}1 / k_{1} \\
1 / k_{2} \\
\text { TT } \\
\text { Temps de séjour }\end{array}$ & $\begin{array}{r}19,1 \pm 4,2 \\
4,9 \pm 1,8 \\
1,5 \pm 0,3\end{array}$ & $\begin{array}{c}29,2 \pm 12,7^{*} \\
8,0 \pm 2,5 \\
1,5 \pm 0,7\end{array}$ & $\begin{array}{r}21,5 \pm 2,9 \\
5,5 \pm 2,9 \\
0,7 \pm 0,3\end{array}$ & $\begin{array}{c}27,1 \pm 4,9^{* *} \\
4,9 \pm 1,5 \\
0,7 \pm 0,3\end{array}$ & $\begin{array}{r}22,2 \pm 2,7 \\
3,5 \pm 1,2 \\
0,7 \pm 0,4\end{array}$ & $\begin{array}{r}39,1 \pm 9,9 \\
4,3 \pm 1,5 \\
0,6 \pm 0,5\end{array}$ \\
\hline total $(1)$ & $24,4 \pm 5,6$ & $38,8 \pm 14,6$ & $27,8 \pm 4,0$ & $32,7 \pm 5,9$ & $26,2 \pm 2,1$ & $44,0 \pm 9,7$ \\
\hline
\end{tabular}

Signification statistique : $* \mathrm{P}<0,05 ; * * \mathrm{P}<0,01$; ( $\left.{ }^{1}\right)$ Temps de séjour total $: \frac{1}{\mathrm{k}_{1}}+\frac{1}{\mathrm{k}_{2}}+\mathrm{TT}$

La cause des différences entre marqueurs est donc à localiser au niveau du rumen. Bien que l'activité microbienne n'ait qu'un rôle mineur dans le phénomène de "comminution " des particules alimentaires (Mc. Burney et al., 1981), il est possible que la vitesse de réduction de la taille des particules mordancées indigestibles soit ralentie, expliquant en partie les différences importantes entre marqueurs observées avec le foin et la luzerne. La densité supérieure des petites particules d'aliment mordancé pourrait également être la cause d'une rétention sélective. Par ailleurs, la possibilité d'une migration des TR sur les particules de petite taille n'est pas à exclure mais nous avons constaté que le " taux de transfert " de $\mathrm{I}^{\prime 169} \mathrm{Yb}$ incubé in vitro dans du contenu de rumen était très faible $\left(0,002 . \mathrm{h}^{-1}\right)$.

Ainsi, qu'elles soient introduites dans le rumen en solution (Poncet et al., 1986) ou préalablement fixées sur un aliment, les TR conduisent à des estimations du temps de séjour inférieures à celles obtenues avec les fibres mordancées au chrome.

Ellis W. C., Beever D. E., 1984. In : P. M. Kennedy, Techniques in particle size analysis of feed and digesta in ruminants. Can. Soc. Anim. Sci., Edmonton, 167.

Grovum W. L., Williams V. J., 1973. Brit. J. Nutr., 30. 313-329.

Mc Burney M. I., Van Soest P. J., Chase L. E., 1981. J. Sci. Food Agric., 34, 910.

Poncet C., Beaufort M. T., AL ABD A., 1986. Reprod. Nutr. Dévelop., 26, 321-322. 